\title{
COMMENTS
}

Comments are short papers which criticize or correct papers of other authors previously published in the Physical Review. Each Comment should state clearly to which paper it refers and must be accompanied by a brief abstract. The same publication schedule as for regular articles is followed, and page proofs are sent to authors.

\section{In defense of the "tunneling" wave function of the universe}

\author{
Jaume Garriga \\ Center For Theoretical Physics, Laboratory for Nuclear Science and Department of Physics, Massachusetts Institute of Technology, \\ Cambridge, Massachusetts 02139 \\ and IFAE, Departament de Física, Universitat Autònoma de Barcelona, 08193 Bellaterra (Barcelona), Spain
}

Alexander Vilenkin Institute of Cosmology, Department of Physics and Astronomy, Tufts University, Medford, Massachusetts 02155

(Received 10 December 1996)

\begin{abstract}
The tunneling approach to the wave function of the Universe has been recently criticized by Bousso and Hawking who claim that it predicts a catastrophic instability of de Sitter space with respect to pair production of black holes. We show that this claim is unfounded. First, we argue that different horizon size regions in de Sitter space cannot be treated as independently created, as they contend. And second, the WKB tunneling wave function is not simply the "inverse" of the Hartle-Hawking one, except in very special cases. Applied to the related problem of pair production of massive particles, we argue that the tunneling wave function leads to a small constant production rate, and not to a catastrophe as the argument of Bousso and Hawking would suggest. [S0556-2821(97)06616-2]

PACS number(s): 98.80.Hw, 04.60.Kz, 04.62.+v
\end{abstract}

\section{INTRODUCTION}

The tunneling proposal for the wave function of the Universe has been recently criticized by Bousso and Hawking [1] who argue that it predicts a catastrophic instability of de Sitter space with respect to production of black hole pairs. Here it will be shown that their argument is incorrect.

Let us first review the Bousso-Hawking argument. First, they estimate the pair production rate for black holes $(\mathrm{BH})$ in an inflating universe as

$$
\Gamma \propto \exp \left[-S_{\mathrm{BH}}+S_{0}\right]
$$

where $S_{0}$ and $S_{\mathrm{BH}}$ are Euclidean actions for de Sitter and (degenerate) Schzwarzschild-de Sitter instantons, respectively. The Schwarzschild-de Sitter instanton, which has the topology of $S_{2} \times S_{2}$, is interpreted [2] as describing nucleation of two black holes whose horizon radii are equal to the radius of the de Sitter horizon, $H^{-1}$. A simple calculation gives (in Planck units)

$$
\Gamma \propto \exp \left(-\pi / 3 H^{2}\right) .
$$

For $H \ll 1$, black hole nucleation is exponentially suppressed. Evaluation of the tunneling rates using instanton techniques is a standard practice in the literature, and results similar to Eqs. (1) and (2) for black hole nucleation have been previously derived by Mellor and Moss [3] and others [4]. We have no objections against these results [5].

Now, Bousso and Hawking suggest that instead of using the standard instanton approach, the nucleation rate can be calculated directly from the wave function of the Universe. They argue that each horizon volume in an inflating universe can be regarded as having been nucleated independently of other horizon regions. The rate of black hole production can then be found as

$$
\Gamma \propto P_{\mathrm{BH}} / P_{0},
$$

where $P_{\mathrm{BH}}$ is the probability of quantum nucleation for a horizon-size universe containing a pair of black holes, and $P_{0}$ is the same without black holes.

The nucleation probability in quantum cosmology is sensitive to the choice of boundary conditions for the wave function of the Universe. With Hartle-Hawking $(\mathrm{HH})$ boundary conditions, the wave function is given by the integral [6]

$$
\psi_{\mathrm{HH}}=\int e^{-S}
$$

where $S$ is the Euclidean action and the integration is taken over compact Euclidean geometries and matter fields with a specified field configuration at the boundary. The boundary configuration is chosen to be the three-geometry at the moment of nucleation. For a de Sitter universe this is the maximal $S_{3}$, while for black holes in de Sitter it is $S_{1} \times S_{2}$. The probability is found from $P \propto|\psi|^{2}$. Assuming that the severe divergence problems of the integral (4) are somehow resolved, one can expect that the dominant contribution to Eq. (4) is given by the instantons

$$
\psi_{\mathrm{HH}} \propto e^{-S}
$$


so that $P \propto e^{-2 S}$, where $2 S$ is the corresponding instanton action. Then Eq. (3) reduces to Eqs. (1) and (2). This completes the quantum-cosmological derivation of the black hole pair production rate.

Turning to the tunneling wave function, Bousso and Hawking argue that, since it is suppressed rather than amplified under the barrier, this wave function should be given by

$$
\text { ' " } \psi_{T},, \propto e^{+S} .
$$

Then the sign of the exponent in Eq. (1) is changed to the opposite, and Eq. (2) is replaced by $\Gamma \propto \exp \left(+\pi / 3 H^{2}\right)$. This shows no suppression of black hole production, indicating an instability of de Sitter space.

We have two objections to this argument. First, we disagree that horizon volumes in an inflating universe can be treated as nucleating independently. Second, the tunneling wave function is not given by Eq. (6), except in some very special cases.

In the next section we explain why we believe that different horizon volumes cannot be regarded as nucleating independently. In Sec. III we discuss the tunneling boundary condition for $\psi$ and explain why it is very difficult to implement it in the problem of black hole nucleation. Fortunately, the tunneling wave function can be found in a very similar problem of massive particle production during inflation. The Bousso-Hawking argument can be applied to this problem without change. If the argument were true, the tunneling wave function would predict a catastrophic instability of de Sitter space with respect to particle production. However, we show in Sec. IV that it actually predicts a small, constant pair production rate, in agreement with instanton calculations.

\section{BLACK HOLE NUCLEATION VS NUCLEATION OF UNIVERSES WITH BLACK HOLES}

To see why different horizon volumes cannot be regarded as nucleated independently, consider a generic case of inflation driven by a scalar field $\phi$ with a slowly varying potential $V(\phi)$, disregarding for the time being the process of black hole production. The field $\phi$ is approximately constant on the horizon scale, and nucleation of horizon-size universes with different values of $\phi$ can be approximately described by de Sitter instantons. The corresponding probability is

$$
P(\phi) \propto \exp \left[ \pm \pi / H^{2}(\phi)\right],
$$

where $H^{2}(\phi)=8 \pi V(\phi) / 3$ and the upper and lower signs are for the Hartle-Hawking and tunneling wave functions, respectively [6-8]. (This is one example when the two wave functions do differ mainly by the sign in the exponent.) Suppose an observer occupies a horizon-size region with $\phi=\phi_{0}$ and wants to know the probability distribution for $\phi$ in the adjacent regions. If different horizon volumes were nucleated independently, then this distribution would be given by Eq. (7) and would be independent of $\phi_{0}$. This would imply rapid variation of the field $\phi$ from one horizon region to another. Of course, the observer will not be able to see this variation as long as inflation continues. But after inflation, all these horizon volumes will eventually come within the observer's horizon, and rapid variation of $\phi$ will manifest itself in large density fluctuations on all scales. This conclusion is in a sharp disagreement with the standard analysis of density fluctuations in the inflationary universe [9].

The evolution of the field $\phi$ during inflation is determined by the quantum state of the field. It is usually assumed that this state is close to the de Sitter-invariant (Bunch-Davies) vacuum. Then, it has been shown [10] that the evolution of $\phi$ can be pictured as a diffusion process, resulting in strong correlations between the values of $\phi$ in adjacent horizon regions. As a consequence, density fluctuations are small on sufficiently small scales. It is possible that some highly excited state of the field would result in evolution suggested by the random nucleation picture. Hence, the quantumcosmological problem of finding the probability for the universe to nucleate with a given value of $\phi$ is not the same as the problem of finding the probability distribution for $\phi$ within the universe. Quantum cosmology can be said to determine the latter distribution only in the sense that it determines the initial quantum state of $\phi$ at the nucleation of the universe.

Quite similarly, the probability of black hole pair production in an inflating universe is determined by the quantum state of the gravitational field. It is not related in any simple way to the probability for the universe to nucleate with a pair of black holes. Equation (3) with the Hartle-Hawking wave function $\psi_{\mathrm{HH}}$ correctly gives the leading exponent of the pair production rate $\Gamma$ only because $\psi_{\mathrm{HH}}$ is defined in terms of the same Euclidean path integrals used in the instanton evaluation of $\Gamma$.

\section{THE TUNNELING WAVE FUNCTION}

The wave function of the Universe $\psi$ is defined on superspace, which is the space of all three-geometries (and matter field configurations). It satisfies the Wheeler-DeWitt equation

$$
\mathcal{H} \psi=0,
$$

where $\mathcal{H}$ is a differential operator on superspace. The tunneling boundary condition, as defined in Refs. [8,11], requires that $\psi$ includes only outgoing waves at singular boundaries of superspace. The physical meaning of this condition is that the Universe originates in a nonsingular way (at the regular boundary), but may end at a singularity. The regular boundary of superspace includes all singular three-geometries which can be obtained as nondegenerate slices of smooth Euclidean four-geometries.

The word "nondegenerate" means that the presence and the nature of the singularity are stable with respect to small perturbations of the slicing. To give an example of a degenerate slicing, consider a torus lying on a horizontal surface and imagine slicing it with horizontal planes. The slice at the bottom is a circle. But if the torus is slightly tilted, the circular slice disappears, and the bottom slice is an isolated point. The circular slice is degenerate in the sense that it is present only for a very special slicing. A rigorous definition of nondegenerate slices is given in Morse theory [12,13]. It can be shown that singularities on nondegenerate slices always occur at isolated points. 
In addition to the tunneling boundary condition, one has to impose some kind of regularity condition, e.g., $|\psi|<\infty$.

We do not know how to solve the Wheeler-DeWitt equation (8), except in simple models of high symmetry, when the infinite number of degrees of freedom of the Universe can be reduced to one or two, with the remaining degrees of freedom being treated as small perturbations. For example, the nucleation of an inflating universe (without black holes) can be described by treating the radius of the Universe, $a$, as a nonperturbative variable and deviations from spherical symmetry as perturbations. The regular boundary of superspace is then at $a=0$, with finite amplitudes of the perturbations.

To find the tunneling wave function for a Universe with a pair of black holes, one needs to construct an appropriate minisuperspace model. The $S_{2} \times S_{2}$ instanton and the symmetry of the problem suggest a minisuperspace consisting of three-geometries having topology $S_{1} \times S_{2}$ with the radius of the sphere $S_{2}$ fixed at $H^{-1}$. This model would have the radius $r$ of the circle $S_{1}$ as its only variable and would certainly be simple enough to solve. However, the minisuperspace boundary at $r=0$ cannot be regarded as a regular boundary. This is clear from the fact that this singular geometry has singularities on a two-dimensional surface $\left(S_{2}\right)$, rather than at an isolated point [14]. A more complicated model, where the radii of $S_{1}$ and $S_{2}$ are both allowed to vary, has a similar problem. The tunneling boundary condition cannot be implemented in such models. Moreover, it appears that such models are not suitable as minisuperspaces, since they include configurations which are unstable with respect to small perturbations. Extensions of $S_{1} \times S_{2}$ models that would not have this problem are bound to be less symmetric and far more difficult to solve. We note that even if we managed to construct a suitable minisuperspace model and solve for the tunneling wave function $\psi_{T}$, this result would be relevant only for the problem of the nucleation of a universe with a pair of black holes, and not for the rate of black hole production during inflation.

\section{NUCLEATION OF MASSIVE PARTICLES}

Instead of trying to fix the $S_{1} \times S_{2}$ minisuperspace for black hole nucleation, we shall consider a related problem of nucleation of massive particles. The corresponding instanton is the de Sitter $S_{4}$ with a particle world line in the form of a big circle [15]. A similar instanton for nucleation of small extremal black holes was discussed by Mellor and Moss [3]. The instanton action is $S_{p}=S_{0}+2 \pi H^{-1} m$, and the nucleation rate is

$$
\Gamma \propto \exp \left[-S_{p}+S_{0}\right]=\exp (-2 \pi m / H)
$$

Particle nucleation in de Sitter space has all relevant features of black hole nucleation, and the Bousso-Hawking argument can be applied to it without change. If the argument were true, then the tunneling wave function would predict a catastrophic instability of de Sitter space with respect to pair production. However, we will show that in fact it gives the correct particle production rate (9).

We shall assume that the mass of the created particles is sufficiently small that their gravitational back reaction can be ignored, $m \ll 1$, yet sufficiently large that the concept of particle can be unambiguously defined in our expanding background. As we shall see, this requires $m \gg H$. This is actually the same mass range in which the world line instanton approximation is valid.

Within this regime, we can represent the particles as excitations of a massive scalar field $\phi$. Let us expand the field configuration as $\phi=\sum f_{n} Q_{n}$, where $Q_{n}$ are the harmonics on the three-sphere. Then the Wheeler-DeWitt equation (8) can be solved perturbatively in $f_{n}$ using the WKB ansatz $\psi \sim \exp (i S)$, where [16]

$$
S=S_{0}(a)+\frac{1}{2} \sum S_{n}(a) f_{n}^{2}
$$

and

$$
\frac{d S_{0}}{d a}= \pm a\left(a^{2} H^{2}-1\right)^{1 / 2}
$$

The solution of the Hamilton-Jacobi equation (11) is the action along the classical de Sitter solution, which represents an inflationary universe. For $a<H^{-1}$ classical motion is forbidden, $S_{0}$ is imaginary, and $\psi$ is a linear combination of growing and decaying exponentials. For $a>H^{-1}$ classical motion is allowed and $\psi$ is a linear combination of incoming and outgoing waves, whose flux points towards and away from the forbidden region, respectively. The tunneling boundary condition at $a \rightarrow \infty$ requires that only the outgoing wave should be present. The matching conditions for $\psi$ then imply that the growing and decaying components have comparable magnitude near the classical turning point $a=H^{-1}$.

With our ansatz, the Wheeler-DeWitt equation reduces to the functional Schrodinger equation for the quantum field $\phi$ in a fixed de Sitter background, with the scale factor playing the role of time. Its solution is given by $S_{n}=H^{2} a^{2} \dot{\nu}_{n} / \nu_{n}$, where a dot indicates derivative with respect to the conformal time $\tau$, defined by $a=(H \cos \tau)^{-1}$, and $\nu_{n}(\tau)$ are normal modes of the classical scalar field equation. For each $n$ there are two independent solutions, and the quantum state is specified once we choose a particular linear combination of these as our "negative frequency" modes $\nu_{n}$. The mode functions $\nu_{n}$ should, in principle, be determined by the outgoing-wave and regularity conditions at the boundaries of superspace: $a \rightarrow 0, f_{n} \rightarrow \pm \infty$. This, however, cannot be implemented within the perturbative approach, since the expansion (10) breaks down at large values of $f_{n}$. Instead, we shall follow Ref. [17] and require that the wave function does not increase towards large $f_{n}$. It appears that this is the best one can do to represent the boundary condition $\psi\left(\left|f_{n}\right| \rightarrow \infty\right)<\infty$. Mathematically, our condition requires that $\operatorname{Im}\left(S_{n}\right)>0$ along the three branches of the semiclassical wave function (growing, decaying, and outgoing). It can be shown that this requirement uniquely determines $\nu_{n}$, and hence the quantum state of the scalar field [17]. The corresponding eigenmodes are given by

$$
\begin{gathered}
\nu_{n} \propto \cos \tau e^{i n \tau} F(\mu, 1-\mu, n+1 ;(1+i \tan \tau) / 2), \\
\mu=\frac{1}{2}-\left(\frac{9}{4}-\frac{m^{2}}{H^{2}}\right)^{1 / 2},
\end{gathered}
$$


where $\tau$ ranges from 0 to $\pi / 2$ for the outgoing branch and from 0 to $\pm i \infty$ for the growing and decaying exponentials. These modes correspond to the Bunch-Davies or de Sitterinvariant vacuum.

The Hartle-Hawking wave function for this model includes only the growing exponential for $a<H^{-1}$ and a linear combination of incoming and outgoing waves with equal amplitudes at $a>H^{-1}$. The mode functions $\nu_{n}$ are given by Eq. (12) and are the same as those for the tunneling wave function. It is easily seen that the two wave functions are not related by the transformation $S \rightarrow-S$, as suggested by Bousso and Hawking [see Eqs. (5), (6)].

It is well known that a particle detector responds in the Bunch-Davies vacuum as if there was a thermal distribution of particles with temperature $H / 2 \pi$ [18]. This is already in qualitative agreement with the instanton result (9). The correspondence can be made even more precise by using the method of Bogoliubov coefficients [20].

For $m \gg H$ and for each $n$, an unambiguous definition of particles can be given at late times. The scalar wave equation is given by

$$
\ddot{\nu}_{n}+3 H \tanh (H t) \dot{\nu}_{n}+m^{2} \nu_{n}+\frac{\left(n^{2}-1\right)}{a^{2}} \nu_{n}=0,
$$

where now the dots indicate derivative with respect to cosmological time defined by $a=\cosh (H t)$. For $t \gg t_{n}$, where $t_{n}$ is the time at which the particles become nonrelativistic, $a\left(t_{n}\right) \sim n H / m$, the last term can be ignored and we have approximate solutions of the form

$$
\nu_{n} \propto a^{-3 / 2}\left(\alpha_{n} e^{i \omega t}+\beta_{n} e^{-i \omega t}\right),
$$

where $\omega \equiv H|\mu-1 / 2| \approx m$. For large $m$, the exponentials oscillate fast compared with the expansion rate and we have a good definition of positive and negative frequency modes corresponding to our "out" vacuum. The Bogoliubov coefficients $\beta_{n}$ can be readily found by using the expansion of the hypergeometric functions (12) at late times and the normalization condition $\left|\alpha_{n}\right|^{2}-\left|\beta_{n}\right|^{2}=1$. The expectation value of the number of "out" particles in a given mode is then

$$
N_{n}=\left|\beta_{n}\right|^{2}=\left(e^{2 \pi \omega / H}-1\right)^{-1} \approx e^{-2 \pi m / H} .
$$

The distribution is independent of $n$, as expected for nonrelativistic particles. At any given time, the occupation numbers of the relativistic modes are exponentially suppressed with respect to the nonrelativistic ones, so the distribution is cut off at $n \sim a m$. The resulting particle production rate per unit physical volume is $(d N / d t d V) \sim m^{3} H \exp (-2 \pi m / H)$ [20]. In Ref. [19] it was shown that the same rate can be obtained by considering the one-loop prefactor to the instanton contribution. There, the cutoff arises because particles with $n \gg a(t) m$ have not been created yet at time $t$.

Hence, the tunneling boundary condition does not lead to disastrous pair production, but to a result which is in good agreement with the instanton calculation.

In following this tunneling vs Hartle-Hawking debate, the reader should be aware that both wave functions are far from being rigorous mathematical objects with clearly specified calculational procedures. Except in the simplest models, the actual calculations of $\psi_{T}$ and $\psi_{\mathrm{HH}}$ involve additional assumptions which appear reasonable, but are not really well justified. (The results presented in this paper are no exception.) For a recent discussion of the problems associated with defining and interpreting the cosmological wave function see, e.g., Ref. [11].

\section{ACKNOWLEDGMENTS}

We are grateful to Serge Winitzki for helpful discussions. This work was partially supported by NATO under Grant No. CRG 951301, by the U.S. Department of Energy under cooperative research agreement DE-FC02-94ER40818, and by the National Science Foundation.
[1] R. Bousso and S. W. Hawking, Phys. Rev. D 54, 6312 (1996); gr-qc/9608009.

[2] P. Ginsparg and M. J. Perry, Nucl. Phys. B222, 245 (1983).

[3] F. Mellor and I. Moss, Phys. Lett. B 222, 361 (1989).

[4] R. B. Mann and S. F. Ross, Phys. Rev. D 52, 2254 (1995).

[5] It should be noted, however, that application of Euclidean methods in de Sitter space does not yet have a satisfactory justification. This was recently emphasized by A. D. Linde, Nucl. Phys. B372, 421 (1992).

[6] J. B. Hartle and S. W. Hawking, Phys. Rev. D 28, 2960 (1983); S. W. Hawking, Nucl. Phys. B239, 257 (1984).

[7] A. D. Linde, Lett. Nuovo Cimento 39, 401 (1984); Ya. B. Zeldovich and A. A. Starobinsky, Sov. Astron. Lett. 10, 135 (1984); V. A. Rubakov, Phys. Lett. 148B, 280 (1984); A. Vilenkin, Phys. Rev. D 30, 509 (1984).

[8] A. Vilenkin, Phys. Rev. D 33, 3560 (1986); 37, 888 (1988).

[9] For a review of inflation, see, e.g., A. D. Linde, Particle Physics and Inflationary Cosmology (Harwood Academic, Chur, Switzerland, 1990).
[10] A. Vilenkin, Phys. Rev. D 27, 2848 (1983); A. D. Linde, Phys. Lett. B 175, 395 (1986); A. A. Starobinsky, in Current Topics in Field Theory, Quantum Gravity and Strings, edited by H. J. de Vega and N. Sanchez (Springer, Heidelberg, 1986).

[11] A. Vilenkin, Phys. Rev. D 50, 2581 (1994).

[12] The importance of using nondegenerate slices has been emphasized in Ref. [11]. For a review of Morse theory see, e.g., J. Milnor, Lectures on h-Cobordism Theorem (Princeton University Press, Princeton, NJ, 1965).

[13] Slices of a four-geometry $\mathcal{M}$ can be obtained as surfaces $f(x)=$ const, where $f(x)$ is a smooth real function on $\mathcal{M}$. These slices will have smooth geometries, except the slices passing through critical points where $\partial_{\mu} f=0$. The latter slices have singular geometries. A singular slice is nondegenerate if $\operatorname{det}\left[\partial_{\mu} \partial_{\nu} f(x)\right] \neq 0$ at the corresponding critical point(s).

[14] The corresponding slicing of $S_{2} \times S_{2}$ can be obtained by choosing $f(x)=\cos \theta$, where $\theta$ is the polar angle on one of the $S_{2}$. Then, $\operatorname{det}\left[\partial_{\mu} \partial_{\nu} f(x)\right]=0$ everywhere, and the singular slices must be degenerate (see the preceding Ref. [13]). 
[15] R. Basu, A. H. Guth, and A. Vilenkin, Phys. Rev. D 44, 340 (1991).

[16] J. J. Halliwell and S. W. Hawking, Phys. Rev. D 31, 1777 (1985).

[17] T. Vachaspati and A. Vilenkin, Phys. Rev. D 37, 898 (1988).
[18] G. W. Gibbons and S. W. Hawking, Phys. Rev. D 15, 2738 (1977).

[19] J. Garriga, Phys. Rev. D 49, 6327 (1994); 49, 6343 (1994).

[20] The Hartle-Hawking wave function gives the same particle production rate, since it predicts the same quantum state of the scalar field. 